\title{
TECTONICALLY-INDUCED DIVERGENCES OF EARTH ROTATION SERIES
}

\author{
R. S. Gross and J. A. Steppe \\ Jet Propulsion Laboratory \\ California Institute of Technology \\ 4800 Oak Grove Drive \\ Pasadena, CA 91109-8099 \\ USA
}

\begin{abstract}
The stability of the Terrestrial Reference Frame (TRF) is studied by analyzing divergences of independently determined Earth rotation series. These series, which have been (nominally) determined within the same TRF, are found to differ by up to $4.7 \mathrm{mas}$ in bias and up to $0.80 \mathrm{mas} / \mathrm{yr}$ in rate. These biasrate differences could reflect errors in the various models used during the data reduction process.
\end{abstract}

\section{Introduction}

A Terrestrial Reference Frame (TRF) can be defined as a right-handed Cartesian reference frame that has been tied to the solid Earth in some prescribed manner (e.g., Kovalevsky et al., 1989). It is realized in practice by specifying the coordinates of a number of globally distributed observing stations. On a tectonically active body such as the Earth, it is not sufficient to just specify the static location of each station, but the vector velocity of each site must be specified as well. As the tectonic plates move about, the positions of the stations located upon them will change. This change in station position must be taken into account when defining the TRF in order for it to be stable and not drift with the stations.

The locations of the observing stations are principally determined by the modern space-geodetic techniques of Very Long Baseline Interferometry (VLBI), Satellite Laser Ranging (SLR), and Lunar Laser Ranging (LLR). In addition (e.g., Lambeck, 1988), these techniques can determine the orientation of the Earth in space, as well as the locations of the laser reflectors (in the case of SLR and LLR), or the locations of the radio sources (in the case of VLBI). Each such solution for the time-dependent station locations and/or Earth rotation parameters defines its own Terrestrial Reference Frame. These solution-specific TRFs can be offset from each other (exhibit differences in bias) and can drift away from each other (exhibit differences in rate) even though each analysis center has followed the procedures and used the constants recommended by the International Earth Rotation Service (IERS; McCarthy, 1989).

These differences in bias and rate between different realizations of the Terrestrial Reference Frame are probably largely caused by errors in the models used when reducing the data. For example, the rate differences are likely to be largely caused by errors in modeling the plate tectonic-induced motions of the observing stations. The different observing techniques (VLBI, SLR, or LLR) use different sets of observing stations that are located on different subsets of the tectonic plates. If the model describing the motions of the tectonic plates is not accurate, then errors in modeling the plate (and hence station) motions are likely to be different for each set of stations, giving rise to rate differences between the solutions.

Differences in bias and rate between different realizations of the Terrestrial Reference Frame can give rise to differences in bias and rate between different solutions for the Earth rotation parameters that have been determined within the different realizations of the TRF. In this paper, the stability of the Terrestrial Reference Frame is studied by analyzing the divergences of independently determined Earth rotation series. 
TABLE 1. DATA SETS STUDIED

\begin{tabular}{llcrcr}
\hline $\begin{array}{c}\text { DATA SET } \\
\text { NAME }\end{array}$ & $\begin{array}{c}\text { DATA } \\
\text { TYPE }\end{array}$ & $\begin{array}{c}\text { ANALYSIS } \\
\text { CENTER }\end{array}$ & $\begin{array}{c}\text { DATA } \\
\text { SPAN }\end{array}$ & $\begin{array}{c}\text { NUMBER } \\
\text { POINTS }\end{array}$ & $\begin{array}{c}\text { PLATE } \\
\text { MODEL }\end{array}$ \\
\hline LLR (L1707; $\Delta \Phi$, UT0) & & & & & \\
MCDonald 2.7m & LLR & JPL & $4 / 15 / 70-6 / 29 / 85$ & 810 & AM0-2 \\
McDonald LRS & LLR & JPL & $3 / 2 / 85-1 / 27 / 88$ & 35 & AM0-2 \\
McDonald LRS (New Site) & LLR & JPL & $3 / 28 / 88-12 / 9 / 89$ & 20 & AM0-2 \\
CERGA & LLR & JPL & $4 / 7 / 84-12 / 20 / 89$ & 271 & AM0-2 \\
Haleakala & LLR & JPL & $11 / 14 / 84-12 / 17 / 89$ & 105 & AM0-2 \\
TEMPO (90 R 01; T, V) & & & & & \\
CA (12)-Spain (63) & VLBI & JPL & $7 / 18 / 82-8 / 25 / 84$ & 57 & AM0-2 \\
CA (14)-Spain (61) & VLBI & JPL & $9 / 19 / 82-8 / 1 / 87$ & 42 & AM0-2 \\
CA (14)-Spain (63) & VLBI & JPL & $11 / 26 / 79-12 / 10 / 89$ & 142 & AM0-2 \\
CA (15)-Spain (63) & VLBI & JPL & $10 / 4 / 87-7 / 8 / 90$ & 42 & AM0-2 \\
CA (12)-Australia (43) & VLBI & JPL & $7 / 2 / 82-7 / 22 / 84$ & 58 & AM0-2 \\
CA (14)-Australia (42) & VLBI & JPL & $2 / 15 / 83-9 / 20 / 87$ & 34 & AM0-2 \\
CA (14)-Australia (43) & VLBI & JPL & $10 / 28 / 78-3 / 3 / 90$ & 170 & AM0-2 \\
CA (15)-Australia (43) & VLBI & JPL & $11 / 8 / 87-7 / 9 / 90$ & 36 & AM0-2 \\
CDP (EOP.629) & & & & & \\
Multi-Baseline & VLBI & GSFC & $8 / 4 / 79-12 / 29 / 89$ & 700 & AM0-2 \\
Westford-Ft. Davis & VLBI & GSFC & $6 / 25 / 81-1 / 1 / 84$ & 103 & AM0-2 \\
NAVNET (NAVY 1990-2) & & & & & \\
Multi-Baseline & VLBI & USNO & $9 / 11 / 88-2 / 21 / 90$ & 38 & AM0-2 \\
CSR (89 L 02; PMX, PMY) & SLR & U. Texas & $5 / 15 / 76-1 / 3 / 89$ & 970 & AM1-2 \\
& & & & & \\
\hline
\end{tabular}

\section{Approach}

Some particulars about the independently determined Earth rotation data sets chosen for this study are given in Table 1. Each series listed was chosen because it has been obtained, at least nominally in rate, within the same Terrestrial Reference Frame, namely that one determined by applying (without adjustment) the plate tectonic motion model AM0-2 of Minster and Jordan (1978) as a model for the motions of the observing stations. Note that the SLR results chosen were obtained by applying (without adjustment) the plate tectonic motion model AM1-2 of Minster and Jordan (1978). However, these SLR results can be (and have been) analytically corrected to the AM0-2 frame by applying a rate adjustment of -0.52 milliarcseconds per year (mas/yr) to the $\mathrm{x}$-component of polar motion (PMX), and of $-0.24 \mathrm{mas} / \mathrm{yr}$ to the $\mathrm{y}$ component (PMY; IERS Annual Report for 1989, pp. II-26). Also note that the SLR UT1 results were not used in this study due to problems associated with separating this component from the effects of unmodelled forces acting on the satellite causing the node of its orbit to change.

These chosen Earth rotation data sets have been intercompared in order to determine bias and rate corrections needed to be applied to each component of each data set in order for it to agree (in bias and rate) with a combination of all the other data sets. At the same time, scale factors have been determined that need to be applied to the stated uncertainties of each component of each series so that its residual (when differenced with a combination of all other series) has a reduced chi-square of one. This intercomparison has been done in an iterative, round-robin fashion in which each series is compared, in turn, to a combination of all others. The incremental bias-rate corrections and uncertainty scale factors determined 
TABLE 2. ADJUSTMENTS TO DATA SETS

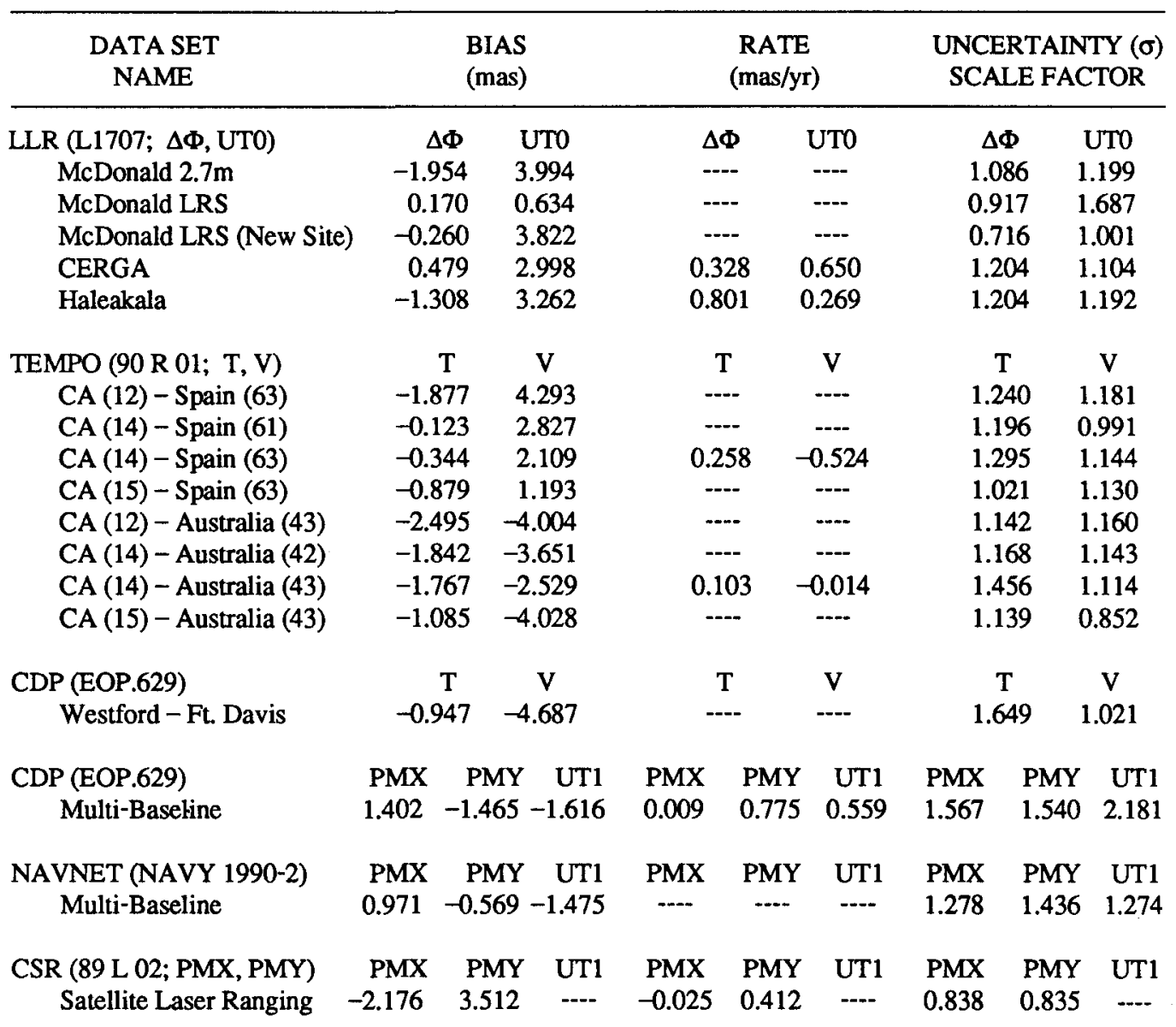

REFERENCE TIME FOR RATE ADJUSTMENT IS 1988.0

during each iteration are applied to the series and the process repeated until convergence is achieved, which is indicated by the incremental bias-rate corrections for each component of each series converging to zero, and the uncertainty scale factors converging to one. Note that rate corrections were obtained only for those series spanning a great enough length of time (and having enough overlap with the other series) that the determination could be reliably obtained.

The combination and intercomparison is done using a Kalman filter approach developed at the Jet Propulsion Laboratory (JPL) for just such a purpose (Eubanks, 1988; Morabito et al., 1988). This approach facilitates comparing and combining data sets of disparate quality, sampling rate, and data type. The comparison is done (and the results reported) in the "natural" reference frame for each data type. For single station LLR results this is the variation of latitude $(\Delta \Phi)$, UT0 frame. For single baseline VLBI results this is the transverse (T), vertical (V) frame (Eubanks and Steppe, 1988). For multi-baseline VLBI and SLR results this is the usual UTPM (PMX, PMY, UT1) frame. 


\section{Results, Discussion, and Conclusions}

The results obtained by this approach are shown in Table 2 which gives the total bias-rate corrections and uncertainty scale factors that must be applied to each component of each raw data set in order for all the data sets to agree with each other. The adjustments to the bias range (in absolute value) up to 4.7 mas, with an average (absolute) value of 2.0 mas and a median (absolute) value of 1.8 mas. The adjustments to the rate range (in absolute value) up to $0.80 \mathrm{mas} / \mathrm{yr}$, with an average (absolute) value of $0.36 \mathrm{mas} / \mathrm{yr}$ and a median (absolute) value of $0.33 \mathrm{mas} / \mathrm{yr}$.

For each baseline of the single baseline VLBI results, the bias adjustment to the vertical component of Earth orientation is larger (in absolute value) than the adjustment to the transverse component. This could be reflecting the property that the vertical component of Earth orientation is more sensitive to errors in the models affecting the local vertical position of the station, such as tropospheric path delay effects, atmospheric and oceanic loading effects, etc. Also note that for each LLR station, the bias adjustment to the UT0 component is greater (in absolute value) than the adjustment to the $\Delta \Phi$ component. These results suggest that modeling errors (of different kinds) are affecting the solution for the Earth rotation parameters in both bias and rate.

Thus, in summary, Earth rotation series that have been determined within the same Terrestrial Reference Frame have been found to differ from each other on average by 2.0 mas in bias and $0.36 \mathrm{mas} / \mathrm{yr}$ in rate. All of the series studied here have been determined by modeling station velocities using a global plate tectonic motion model. This model indicates that the tectonic plates (and hence the stations located upon them) move with respect to each other by up to about 4 mas/yr (Minster and Jordan, 1978). The divergences of the Earth rotation series found in this study are likely to be caused (at least in part) by errors in modeling the motions of the stations. Thus these divergences could perhaps be reduced by applying an improved plate tectonic motion model (such as the NUVEL-1 model of DeMets et al., 1990) or by directly solving for the station velocities (e.g., Ma and Clark, 1990).

ACKNOWLEDGMENTS. The work described in this paper was performed at the Jet Propulsion Laboratory, California Institute of Technology, under contract with the National Aeronautics and Space Administration.

\section{References}

DeMets, C., R. G. Gordon, D. F. Argus, and S. Stein, Current plate motions, Geophys. J. Int., 101, 425-478, 1990.

Eubanks, T. M., Combined Earth orientation series smoothed by a Kalman filter, in Bureau International de l'Heure Annual Report for 1987, pp. D85-D86, Observatoire de Paris, Paris, France, 1988.

Eubanks, T. M., and J. A. Steppe, The long term stability of VLBI Earth orientation measurements, in The Impact of VLBI on Astrophysics and Geophysics, edited by M. J. Reid and J. M. Moran, pp. 369-370, D. Reidel, Dordrecht, Holland, 1988.

Kovalevsky, J., I. I. Mueller, and B. Kolaczek (eds.), Reference Frames in Astronomy and Geophysics, 474 pp., Kluwer, Norwell, Mass., 1989.

Lambeck, K., Geophysical Geodesy: The Slow Deformations of the Earth, 718 pp., Oxford University Press, Oxford, 1988.

Ma, C., and T. A. Clark, NASA Crustal Dynamics Project VLBI Terrestrial and Celestial Reference Frames (abstract), EOS, Trans. Amer. Geophys. Union, 71, 482, 1990.

McCarthy, D. D. (Ed.), IERS Standards (1989), IERS Technical Note 3, 76 pp., Central Bureau of IERS Observatoire de Paris, Paris, France, 1989.

Minster, J. B., and T. H. Jordan, Present-day plate motions, J. Geophys. Res., 83, 5331-5354, 1978.

Morabito, D. D., T. M. Eubanks, and J. A. Steppe, Kalman filtering of Earth orientation changes, in The Earth's Rotation and Reference Frames for Geodesy and Geodynamics, edited by A. K. Babcock and G. A. Wilkins, pp. 257-267, D. Reidel, Dordrecht, Holland, 1988. 